\section{FIBRES AND WHISKERS}

\section{Fibre Science and Technology}

Vol. 1, No. 1, July 1968. Quarterly. (Elsevier: London.) 150s. per annum.

In recent years there have been important developments in the production of carbon fibres and of "whiskers" of various types. These materials are exceptionally strong and when incorporated into plastics yield new products which can often be used to replace metals. Research and development in this field have been associated with ongineering science rather than the science of textile fibres and processing, and it is therefore perhaps understandablo that special provision should be made for the publication of results.

The new journal Fibre Science and Technology provides such a medium. It is edited by distinguished scientists and will be devoted "primarily to fibre and whisker preparation, their structure and related physical properties, their incorporation into a matrix, forming methods and the application of products". The subjects of the original papers in the first issue illustrate clearly the types of investigation which are fittingly included: for example, carbon-fibre reinforced plastics; preparation of silicon carbide-coated boron filaments ; preparation of cast boronaluminium composites; controllable shrinkage properties of fibres; processing particulate composites.

In one sense the new journal will duplicate the facilities offered by such journals as the Textile Research Journal and the Journal of the Textile Institute, but with a new and expanding subject there is room for additional opportunities for publication. If the launching of a new journal encourages engineers and those interested in materials science to become familiar with the work which has been and is being done on "textile fibres", nothing but good can emerge.

The title of the journal is, perhaps, unfortunate, for Fibre Science and Technology embraces a far wider range of topics than those associated with the production of strengthened materials and composites. This is, however, a minor criticism and the many research and development workers in fibre science will look forward to the publication of high quality contributions in an exciting and extremely important field.

C. S. WHEWELI

\section{HIGH TEMPERATURE MATERIALS}

High Temperature Materials

The Controlling Physical Processes. Edited by A. J. Kennedy. Pp. vii +102. (Oliver and Boyd: Edinburgh and London, 1968.) 47s. $6 d$.

IN 1965, Professor Kennedy organized a meeting in London, whose object was to clarify understanding of the factors which limit the engineering use of materials at high temperatures. The five review papers presented at that meeting have been brought up to date, and form, with an introduction by Dr Kennedy, the basis of this new book. These short reviews cover, in order, deformation during creep (D. McLean), structural stability in high temperature materials (C. N. Reid), rate limiting reactions in high temperature oxidation processes (B. B. Argent and N. Birks), brittleness in ceramics (G. D. Miles and F. J. P. Clarke), and elevated temperature failure processes (C. J. Beevers).

Dr McLean's thesis is that recent studies of the threedimensional dislocation networks formed in metals during creep indicate that the steady state creep rate can be interpreted on a recovery theory: the mesh size of the networks is continuously being expanded by recovery and refined by strain-hardening. In multi-phase alloys, the restraint of recovery of networks by dispersed particles is discussed - the growth of particle spacing with time being considered a controlling feature.

This conclusion leads us to Dr Reid's contribution, where he observes that oxide in metal dispersions offers stable creep resistant structures of high dislocation density. This paper consists of a conventional qualitative account of the principal methods of strengthening of metals-by solid solution, by work hardening, by precipitation hardening and oxide dispersion, and their dependence on temperature.

Dr Beevers gives a stimulating review of the high temperature fracture of metals, in particular the role of grain boundary sliding on the nucleation of intergranular failure by processes of wedge cracking or of cavitation. Although the relation between theory and observation is encouraging, considerably more research is clearly nceded before these processes can be understood in detail.

The paper by Miles and Clarke is the only one concerned with non-metals, thereby justifying the use of that fashionable word "materials" in the title of the book. It consists essentially of an authoritative review of the fracture behaviour of magnesium oxide-firstly, the plastic processes leading to the nucleation and growth of brittle cracks in single crystals of magnesia at ambient temperatures, and then the deformation and fracture of single crystals and polycrystals at high temperatures. In these cases, and in the deformation of the other polycrystalline ceramics briefly considered, the mechanical behaviour is shown to be governed by the number and distribution of the available slip systems.

The article on oxidation processes in metals by Professor Argent and Dr Birks has no common ground with the other papers in the book, although it must be faced that oxidation resistance is a key requirement for high temperature materials. It gives a scholarly account of the possible processes involved in the formation of solid films on metals, discussing iron and nickel in particular and also the behaviour of alloys under these conditions. Liquid and gaseous oxides are also briefly considered.

The book itself is well produced and clearly illustrated, and can be recommended for all students of metallurgy and materials science.

J. W. Martin

\section{HYPERFINE STRUCTURE}

\section{Hyperfine Structure and Nuclear Radiations}

Edited by E. Matthias and D. A. Shirley. (Proceedings of a conference held at Asilomar, Pacific Grove, California, USA, August 25-30, 1967.) Pp. xiii $+1,097$. (North-Holland: Amsterdam, 1968.) 1868.

IT is well known that the electric or magnetic coupling between the spin of the nucleus and its surrounding electrons is responsible for the hyperfine structure seen in atomic spectra. In the past twenty years, with the development of refined detection methods the influence of hyperfine structure on nuclear disintegration processes can now be seen and this is rapidly becoming a rewarding method for investigating nuclear structure and for studying fields in solids. Because of the growing interest in this interdisciplinary area a conference was arranged at which were presented nearly 100 papers ranging from reviews to short contributions and these are included verbatim in this volume.

A number of the contributed papers describe Mössbauor measurements of excited state moments and of internal fields in solids. Similar information can also be obtained from the precession of a gamma ray angular correlation caused by coupling with a magnetic field and a number of elegant experiments were reported in which this effect was studied. In some of these the ion-implantation technique was used to embed the excited nuclei in a supporting foil and values of tho internal fields at these 